\title{
Tratamento artroscópico da condromatose sinovial do ombro: relato de caso*
}

\author{
Arthroscopic treatment of synovial \\ chondromatosis of the shoulder: a case report
}

Arildo Eustáouio Paim, DANiel Costa Ferreira², Alessandro Paim², Ricardo Mota de Almeida ${ }^{3}$

\section{RESUMO}

Os autores descrevem caso raro de condromatose sinovial do ombro e seu tratamento cirúrgico. A artroscopia possibilitou a visualização de todos os compartimentos da articulação glenoumeral, permitindo a remoção dos corpos livres e realização da sinovectomia.

Descritores - Condromatose sinovial/cirurgia; Articulação do ombro; Membrana sinovial; Artroscopia; Corpos livres articulares; Relatos de casos [Tipo de publicação]

\section{ABSTRACT}

The authors describe a rare case of synovial chondromatosis of the shoulder and its surgical treatment. Arthroscopy enabled the visualization of

* Trabalho realizado no Grupo de Cirurgia de Ombro e Cotovelo do Serviço de Ortopedia e Traumatologia da Santa Casa de Belo Horizonte - Belo Horizonte (MG), Brasil.

1. Chefe do Grupo de Grupo de Cirurgia de Ombro e Cotovelo da Santa Casa de Belo Horizonte - Belo Horizonte (MG), Brasil.

2. Assistente do Grupo de Grupo de Cirurgia de Ombro e Cotovelo da Santa Casa de Belo Horizonte - Belo Horizonte (MG), Brasil.

3. Residente do 4 ano do Grupo de Grupo de Cirurgia de Ombro e Cotovelo da Santa Casa de Belo Horizonte - Belo Horizonte (MG), Brasil.

Endereço para correspondência: Rua Ramalhete 550/500, Serra 30210-500 - Belo Horizonte, MG. Tel.: (31) 3213-4398.

E-mail: Arildo.bhe@terra.com.br

Recebido em 30/10/07. Aprovado para publicação em 8/4/08.

Copyright RBO2008 all compartment of the glenohumeral joint, and allowed the removal of free bodies and the performance of a synovectomy.

Keywords - Chondromatosis, synovial/surgery; Shoulder joint; Synovial membrane; Arthroscopy; Joint loose bodies; Case reports [Publication type]

\section{INTRODUÇÃO}

A condromatose sinovial primária do ombro é uma situação clínica rara, que cursa com a formação de múltiplos corpos livres intra-articulares nos vários compartimentos da articulação glenoumeral. O presente relato de caso tem como objetivo mostrar que a artroscopia permitiu visualizar todos os compartimentos intra-articulares acometidos, retirar os corpos livres e realizar a sinovectomia.

\section{RELATO DO CASO}

Paciente do sexo feminino, 23 anos de idade, com história de dor no ombro direito havia cerca de um ano, sem história de traumatismo. Durante o exame físico, a paciente apresentava dor à movimentação do ombro e limitação da amplitude de movimento $\left(100^{\circ}\right.$ de elevação anterior, $45^{\circ}$ de rotação externa, rotação interna em T8 e $45^{\circ}$ de abdução) (figura 1).

Estudos por imagem foram obtidos através de radiografias simples e artrorressonância magnética, os quais demonstraram múltiplos corpos livres intra-articulares e osteoartrose glenoumeral incipiente (figura 2). 

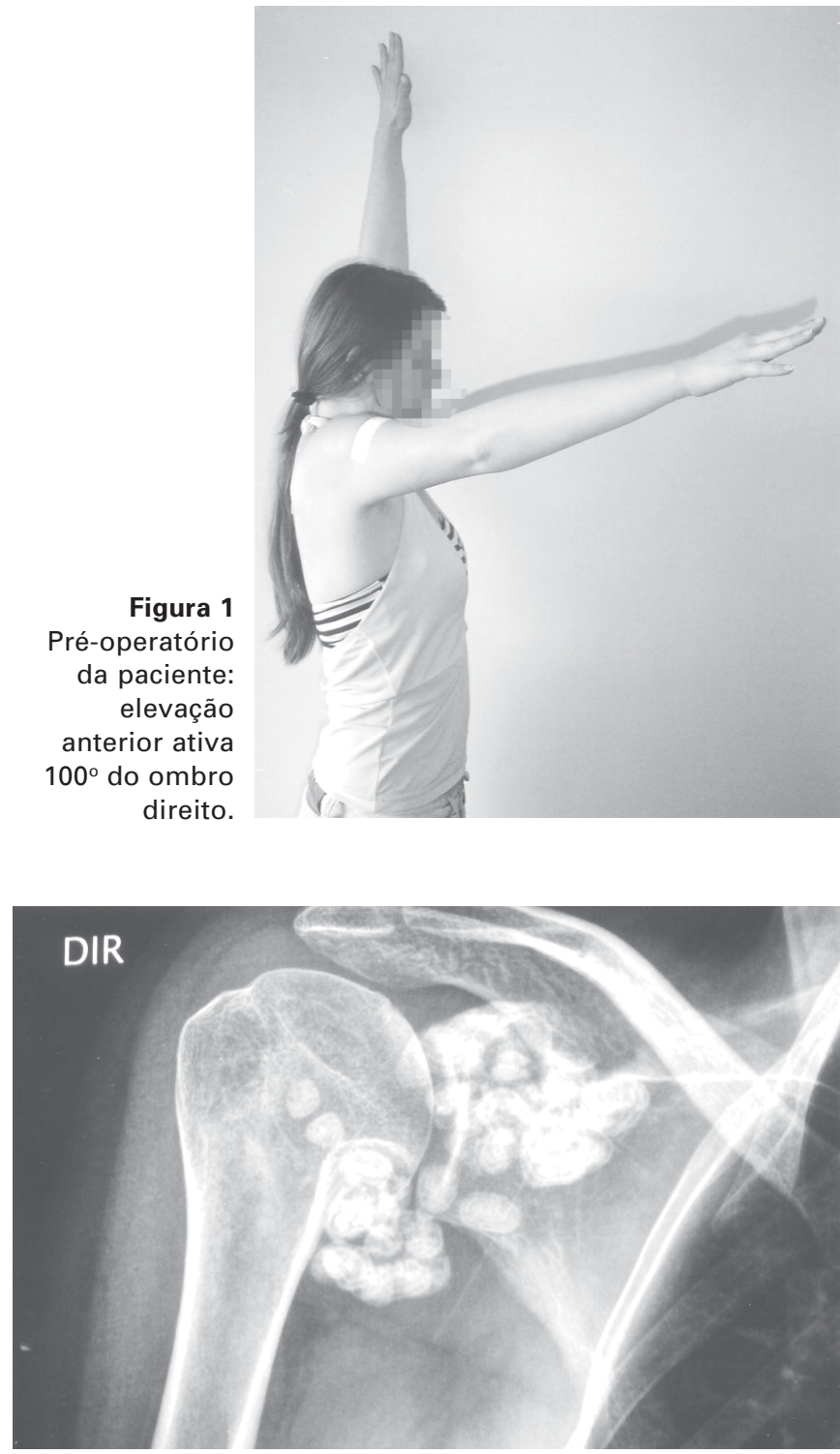

Figura 2 - Radiografia pré-operatória do ombro direito: presença de múltiplos corpos livres e sinais de osteoartrose glenoumeral.

O tratamento cirúrgico foi realizado por via artroscópica, na Santa Casa de Belo Horizonte. Após a realização de bloqueio interescalênico e anestesia geral, a paciente foi posicionada em decúbito lateral. Quatro portais foram feitos, sendo dois posteriores e dois anteriores. Os compartimentos: anterior, posterior, os recessos axilar e subescapular foram visualizados com os corpos livres (figura 3). Durante o ato cirúrgico observamos lesões condrais, tanto na cavidade glenóidea quanto na cabeça umeral. A sinovite estava pre-

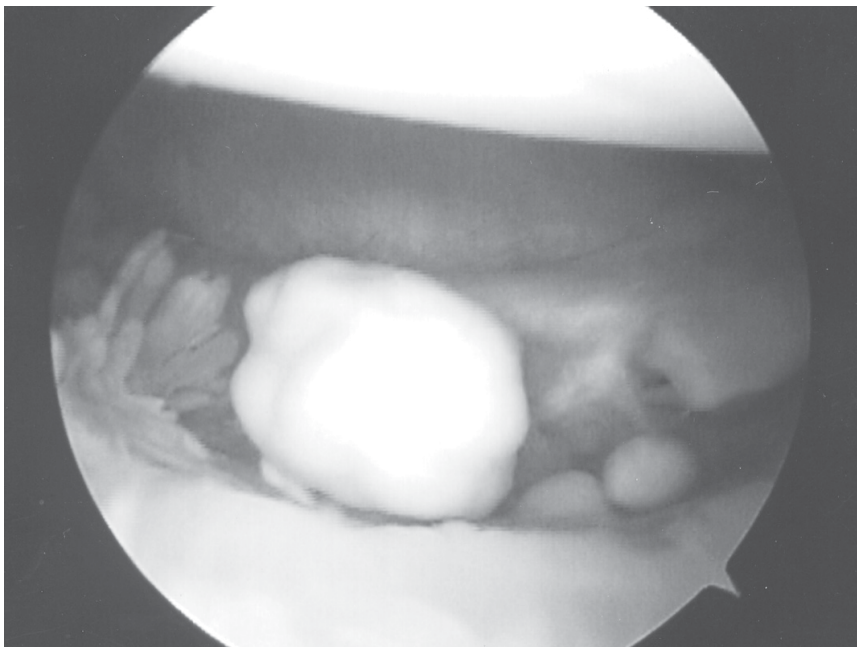

Figura 3 - Peroperatório: visão artroscópica dos corpos livres intra-articulares.

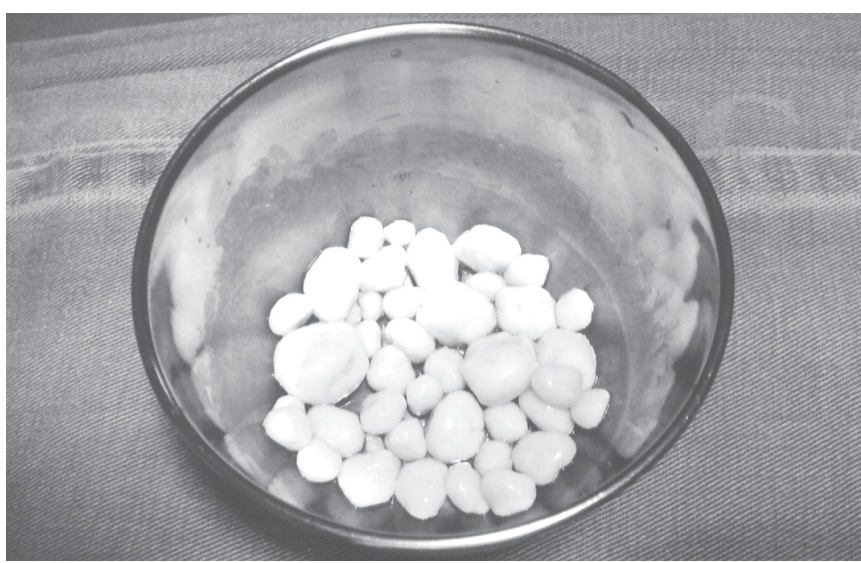

Figura 4 - Pós-operatório: aspecto macroscópico dos corpos livres.

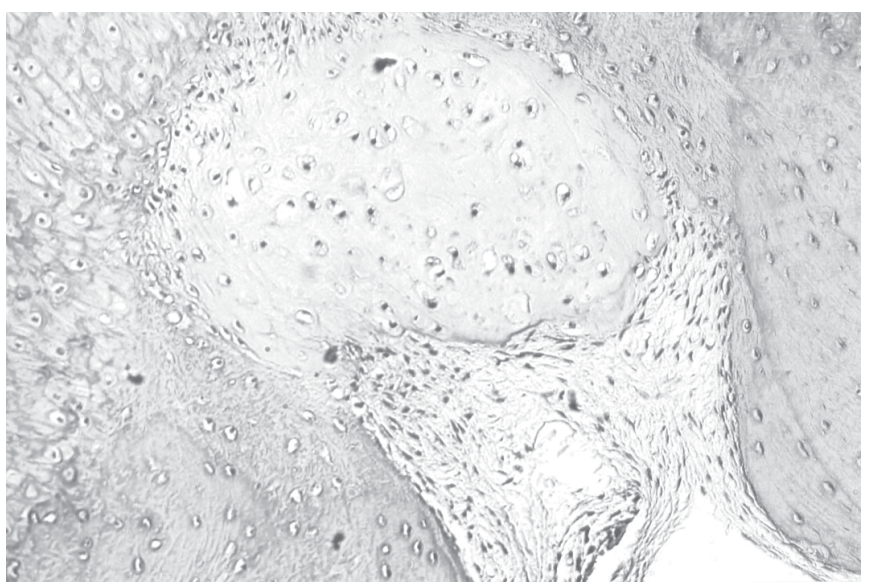

Figura 5 - Corte histológico de corpo livre cartilaginoso calcificado, contendo pequeno nódulo metaplásico imaturo, parcialmente revestido por membrana sinovial (HE, 10x) 


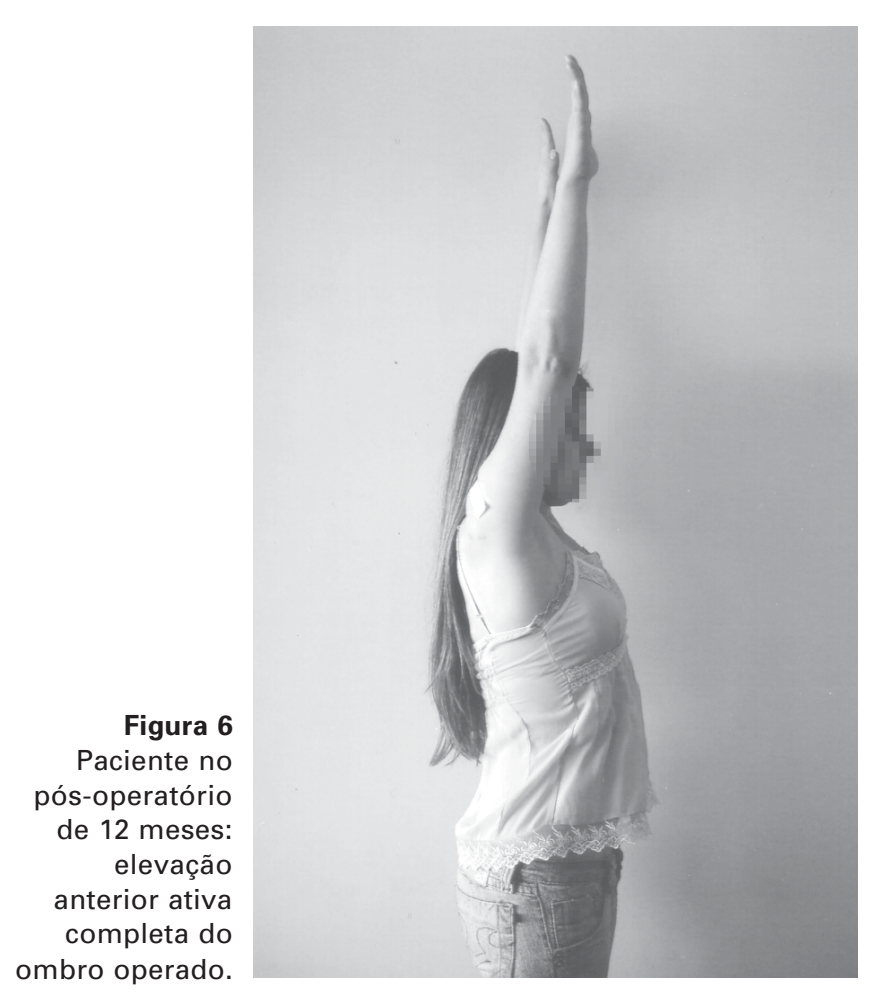

sente em pequenas áreas. Através de cânulas cirúrgicas de $8,25 \mathrm{~mm}$ foi possível retirar 44 corpos livres (figura 4). A sinovectomia foi realizada após a retirada dos corpos livres para evitar sangramento excessivo durante o ato cirúrgico. $\mathrm{O}$ exame histológico revelou corpos livres cartilaginosos calcificados com pequenos nódulos metaplásicos imaturos e revestidos por membrana sinovial caracterizando condromatose sinovial (figura 5).

No pós-operatório, a paciente usou tipóia durante 10 dias, permitindo exercícios autopassivos para o ombro, cotovelo e mão. Depois desse período foi iniciada a fisioterapia formal. Na avaliação após 12 meses da cirurgia, a paciente relatou melhora da dor e retorno da amplitude de movimento do ombro operado (figura 6). O controle radiográfico constatou ausência de corpos livres e não progressão da osteoartrose glenoumeral (figura 7).

\section{DISCUSSÃO}

A condromatose sinovial primária é afecção rara, benigna, normalmente monoarticular, na qual ocorre

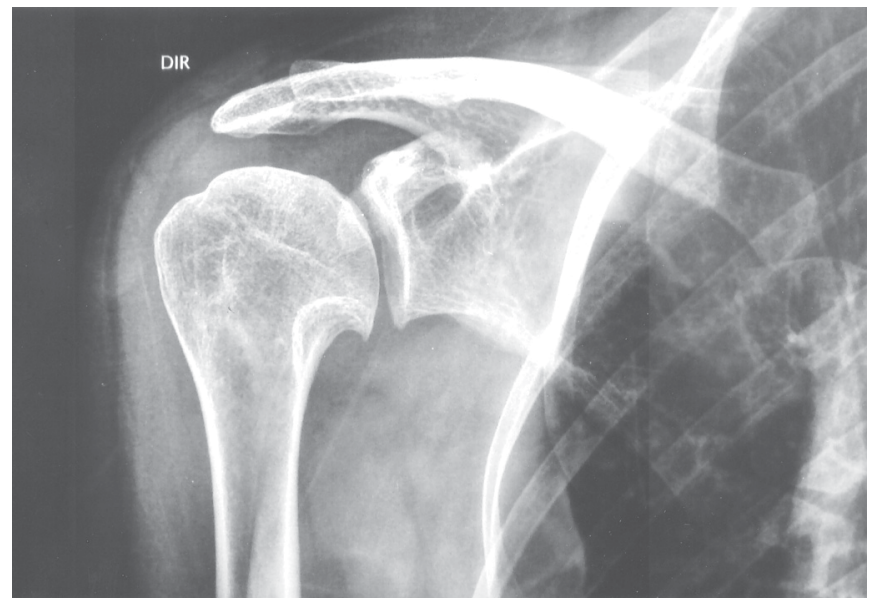

Figura 7 - Radiografia pós-operatória do ombro direito com 12 meses: ausência de corpos livres.

a formação de corpos livres cartilaginosos. Isso se dá, principalmente, por metaplasia da membrana sinovial articular, podendo ocorrer também por metaplasia da bainha de tendões ou, até mesmo, da bursa ${ }^{(1)}$. Quando ocorre em uma articulação previamente acometida por alguma condição patológica, como osteoartrose ou osteonecrose, é denominada condromatose sinovial secundária $^{(1)}$.

A articulação mais acometida é o joelho, seguido do quadril, cotovelo, punho, tornozelo e ombro. Bloom e Pattinson revisaram 191 casos de condromatose sinovial e somente 10 casos (cerca de 5\%) envolviam o ombro $^{(2)}$.

A sintomatologia inclui dor, inchaço, crepitação e limitação do movimento.

A radiografia simples pode ser negativa nos estágios iniciais e a ressonância magnética pode identificar corpos livres em estágios precoces.

Milgram descreveu 30 casos de condromatose sinovial, identificando três estágios distintos: (1) doença com atividade intra-sinovial, mas sem a presença de corpos livres; (2) lesão transicional, com atividade sinovial e corpos livres; (3) múltiplos corpos livres, mas sem atividade sinovial ${ }^{(3)}$. A paciente que estudamos se encontrava no estágio 3 , pois apresentava múltiplos corpos livres intra-articulares e na artroscopia observamos discretos focos de sinovite. 
A transformação maligna pode ocorrer, porém, é mais comum após múltiplos episódios de recidiva. Davis et al revisaram 53 pacientes com condromatose sinovial primária e identificaram recidiva em nove deles $(17 \%)$. Três desses nove pacientes sofreram transformação maligna para condrossarcoma ${ }^{(4)}$.

Apesar dos relatos de remissão espontânea, o tratamento de escolha é o cirúrgico ${ }^{(5)}$. A via tradicional é utilizada para a retirada dos corpos livres e sinovecto$\operatorname{mia}^{(6)}$. Neer recomendou a desinserção do tendão do subescapular e abertura suficiente da cápsula para subluxar a cabeça umeral ${ }^{(5)}$. O advento da artroscopia, além das vantagens como pequenas incisões e reabilitação mais rápida, permite avaliar toda a articulação glenoumeral, com possibilidade de retirada de corpos livres, sinovectomia, tenodese da cabeça longa do bíceps quando comprometida e desbridamento da cartilagem $\operatorname{articular}^{(7-8)}$. Neste caso, através da artroscopia, conseguimos visualizar todos os compartimentos do ombro com apenas quatro pequenas incisões (portais), retirar os corpos livres e realizar a sinovectomia.

A recidiva é pouco freqüente $(0$ a $15 \%)$ e está relacionada à retirada insuficiente dos corpos livres articulares ou sinovectomia incompleta da membrana sinovial patológica ${ }^{(9)}$. Atualmente, a paciente, motivo deste relato, se encontra no período pós-operatório de 12 meses, sem dor, com os movimentos normais do ombro operado sem sinais de recidiva.

\section{COMENTÁRIO}

Na condromatose sinovial do ombro, o tratamento por via artroscópica parece ser indicação terapêutica eficiente.

\section{REFERÊNCIAS}

1. Milgram JW, Hadesman WM. Synovial osteochondromatosis in the subacromial bursa. Clin Orthop Relat Res. 1988;(236):154-9.

2. Bloom R, Pattinson JN. Osteochondromatosis of the hip joint. J Bone Joint Surg Br. 1951;33(1):80-4.

3. Milgram JW. Synovial osteochondromatosis: a histopathological study of thirty cases. J Bone Joint Surg Am. 1977;59(6):792801.

4. Davis RI, Hamilton A, Biggart JD. Primary synovial chondromatosis: a clinicopathologic review and assessment of malignant potential. Hum Pathol. 1998;29(7): 683-8.

5. Neer CS. Rare shoulder lesions: glenoumeral osteochondromatosis. In: Neer CS, editor. Shoulder reconstruction. Philadelphia: Saunders; 1990. p. 478-9.

6. Brasil Filho R, Filardi Filho CS, Menitti EL, Baptista MV, Daher SS. Condromatose sinovial: relato de um caso. Rev Bras Ortop. 1997;32(11):921-3.

7. Snyder SJ. Synovial chondromatosis and osteochondromatosis. In: Snyder SJ. Shoulder arthroscopy. 2nd ed. Philadelphia: Lippincott Williams \& Wilkins; 2003. p. 281-3.

8. Lunn JV, Castellanos-Rosas J, Walch G. Arthroscopic synovectomy, removal of loose bodies and selective biceps tenodesis for synovial chondromatosis of the shoulder. J Bone Joint Surg Br. 2007;89(10): 1329-35.

9. Sah AP, Geller DS, Mankin HJ, Rosemberg AE, Delaney TF, Wright CD, Hornicek FJ. Malignant transformation of synovial chondromatosis of the shoulder to chondrosarcoma. A case report. J Bone Joint Surg Am. 2007;89(6):1321-8. 